\title{
Mutations in DMI3 and SUNN Modify the Appressorium-Responsive Root Proteome in Arbuscular Mycorrhiza
}

\author{
Nardjis Amiour, Ghislaine Recorbet, Franck Robert, Silvio Gianinazzi, and Eliane Dumas-Gaudot \\ Unité Mixte de Recherche Plante-Microbe-Environnement INRA 1088, CNRS 5184, Université de Bourgogne, INRA-CMSE, \\ BP 86510, 21065 Dijon CEDEX, France
}

Submitted 26 October 2005. Accepted 10 April 2006.

\begin{abstract}
Modification of the Medicago truncatula root proteome during the early stage of arbuscular mycorrhizal symbiosis was investigated by comparing, using two-dimensional electrophoresis, the protein patterns obtained from noninoculated roots and roots synchronized for Glomus intraradices appressorium formation. This approach was conducted in wild-type (J5), mycorrhiza-defective (TRV25, dmi3), and autoregulation-defective (TR122, sunn) M. truncatula genotypes. The groups of proteins that responded to appressorium formation were further compared between wild-type and mutant genotypes; few overlaps and major differences were recorded, demonstrating that mutations in $D M I 3$ and $S U N N$ modified the appressorium-responsive root proteome. Except for a chalcone reductase, none of the differentially displayed proteins that could be identified using matrix-assisted laser desorption ionization time-offlight mass spectrometry previously was known as appressorium responsive. A DMI3-dependent increased accumulation of signal transduction-related proteins (dehydroascorbate reductase, cyclophilin, and actin depolymerization factor) was found to precede mycorrhiza establishment. Differences in the accumulation of proteins related to plant defense reactions, cytoskeleton rearrangements, and auxin signaling upon symbiont contact were recorded between wild-type and hypermycorrhizal genotypes, pointing to some putative pathways by which SUNN may regulate very early arbuscule formation.
\end{abstract}

Additional keywords: flavodoxin, GST, MtAnn1, narbonin, protease inhibitor.

Occurring between more than $80 \%$ of vascular land plants and approximately 150 fungal species belonging to the Glomeromycota, arbuscular mycorrhizal (AM) symbiosis is one of the most widely distributed root-microbe interactions (Smith and Read 1997). This mutualistic association is characterized by a bilateral exchange between the two symbionts: plants benefit from an improved mineral nutrient uptake from the soil (mainly phosphorus) while, in turn, AM fungi are supplied with the organic carbon forms essential for achieving their full life cycle (Harrison 1999). The successful development of AM symbiosis is dependent upon significant morphological and physiological modifications affecting both partners and occurring in a time-orchestrated way. Following a typical hyphal branching in response to the recently identified plant

Corresponding author: G. Recorbet; E-mail: recorbet@epoisses.inra.fr strigolactones (Akiyama et al. 2005), the fungus differentiates appressoria at the root surface representing the first cell-to-cell contact between the two symbiotic partners (Giovannetti et al. 1996). Then, the fungus enters the root epidermis and proliferates within the cortical parenchyma where it forms highly branched structures, called arbuscules, which are thought to be the major sites of solute transfer between the two symbionts (Gianinazzi-Pearson 1996).

In contrast to many plant-pathogenic fungus interactions (Tucker and Talbot 2001), very little is known about the molecular events occurring prior to and during the initial contact between the two symbionts. This partly accounts for the fact that AM fungi are not amenable to axenic culturing in the absence of host roots and that mycorrhizal development is an asynchronous process. Using isolated cell walls of carrot roots, Nagahashi and Douds (1997) demonstrated that appressorium formation by AM fungi was a contact recognition event that did not require a signal secreted from the host root or the presence of intact host cells. However, the induction of an $\mathrm{H}^{+}$-ATPase gene in the AM fungus Glomus mosseae upon appressorium formation supports the hypothesis that appressoria may form in response to a plant signal (Requena et al. 2003). Likewise, the activation of plant defense genes that correlated with appressorium development indicated the existence of fungal signal molecules during the early stages of the mycorrhizal symbiosis (Blilou et al. 2000; Ruiz-Lozano et al. 1999). Recently, two suppressive subtractive hybridization (SSH)-based experiments were performed simultaneously to monitor, at a large scale, both fungal and plant genes regulated upon symbiont cell contact. Several G. mosseae genes related to $\mathrm{Ca}^{2+}$-dependent signaling were found among those induced at an early time point of appressorium formation (Breuninger and Requena 2004) and most of the plant genes upregulated prior to Medicago truncatula root penetration by $G$. mosseae belonged to a signal transduction pathway (Weidmann et al. 2004).

In recent years, an overlap in the activation of gene expression and in signal transduction pathways between AM and nitrogen-fixing symbioses also has emerged from the isolation of plant mutants that abolish both early symbiotic responses to rhizobia (Nod ${ }^{-}$phenotype) and mycorrhiza development (Myc ${ }^{-}$phenotype) (Marsh and Schultze 2001). Map-based cloning strategies applied to such mutations in the model legume $M$. truncatula have led to the identification of three "does not make infection" (DMI) genes necessary for both symbioses, including $D M I 3$, which has sequence similarity with calcium- and calmodulin-dependent protein kinase encoding genes (Lévy et al. 2004; Mitra et al. 2004). Although plants mutated for these genes are unable to develop a functional 
interaction with AM fungi, appressoria are able to develop on their roots without penetration (Calantzis et al. 2001; Catoira et al. 2000; Sagan et al. 1995). The fact that nodulation systemically suppresses further mycorrhization and vice versa also has pointed to a common autoregulatory mechanism shared between the two symbioses (Catford et al. 2003; Vierheilig 2004). Recently, the first gene involved in the autoregulation of mycorrhization and nodulation in $M$. truncatula has been identified. Plants mutated for the SUNN/MtSYM12 gene that encodes a putative leucine-rich repeat receptor-like kinase (LRR-RLK) (Schnabel et al. 2005) displayed an increase in mycorrhizal colonization intensity (hypermycorrhizal phenotype) and a higher number of nodules (supernodulating phenotype) when inoculated with AM fungi and rhizobia, respectively (Sagan et al. 1995).

Although proteins are well known as key effectors of plant responses to environmental cues, including recognition, signaling, transport, and defense reactions, very little is known about changes in protein expression in roots upon AM symbiont cell contact. From the time-course analysis of symbiosis-related proteins in G. mosseae-inoculated M. truncatula plants, there is evidence that the root proteome is modified upon appressorium formation (Bestel-Corre et al. 2002). However, none of the appressorium-responsive proteins was identified. In the present work, we have furthered the analysis of the proteins that are differentially displayed during the early stage of AM symbiosis by comparing, using two-dimensional electrophoresis (2-DE), the total protein patterns obtained from noninoculated roots and roots synchronized for $G$. intraradices appressorium formation (Weidmann et al. 2004). This approach was conducted in wildtype (J5), mycorrhiza-defective (TRV25, dmi3), and autoregulation-defective (TR122, sunn) M. truncatula genotypes. In this study, we report for the first time modification of the root proteome upon symbiont contact in three genotypes differing in $\mathrm{AM}$ fungus colonization intensity and identification by mass spectrometry of appressorium-responsive proteins.

\section{RESULTS}

\section{Fungal development.}

To obtain roots synchronized for the appressorium stage, the early development of $G$. intraradices on $M$. truncatula roots was monitored. In all genotypes (J5, TR122, and TRV25), an average of 5 appressoria/cm of root was reached 5 days after inoculation and fungal penetration within roots was never observed in the duplicated experiments performed (data not shown), allowing us to assign changes in protein abundance upon $G$. intraradices inoculation to the preinfection stage.

\section{Comparison of $M$. truncatula root protein maps.}

After 2-DE of total soluble root proteins and silver staining, reproducible protein patterns were obtained between replicates
A

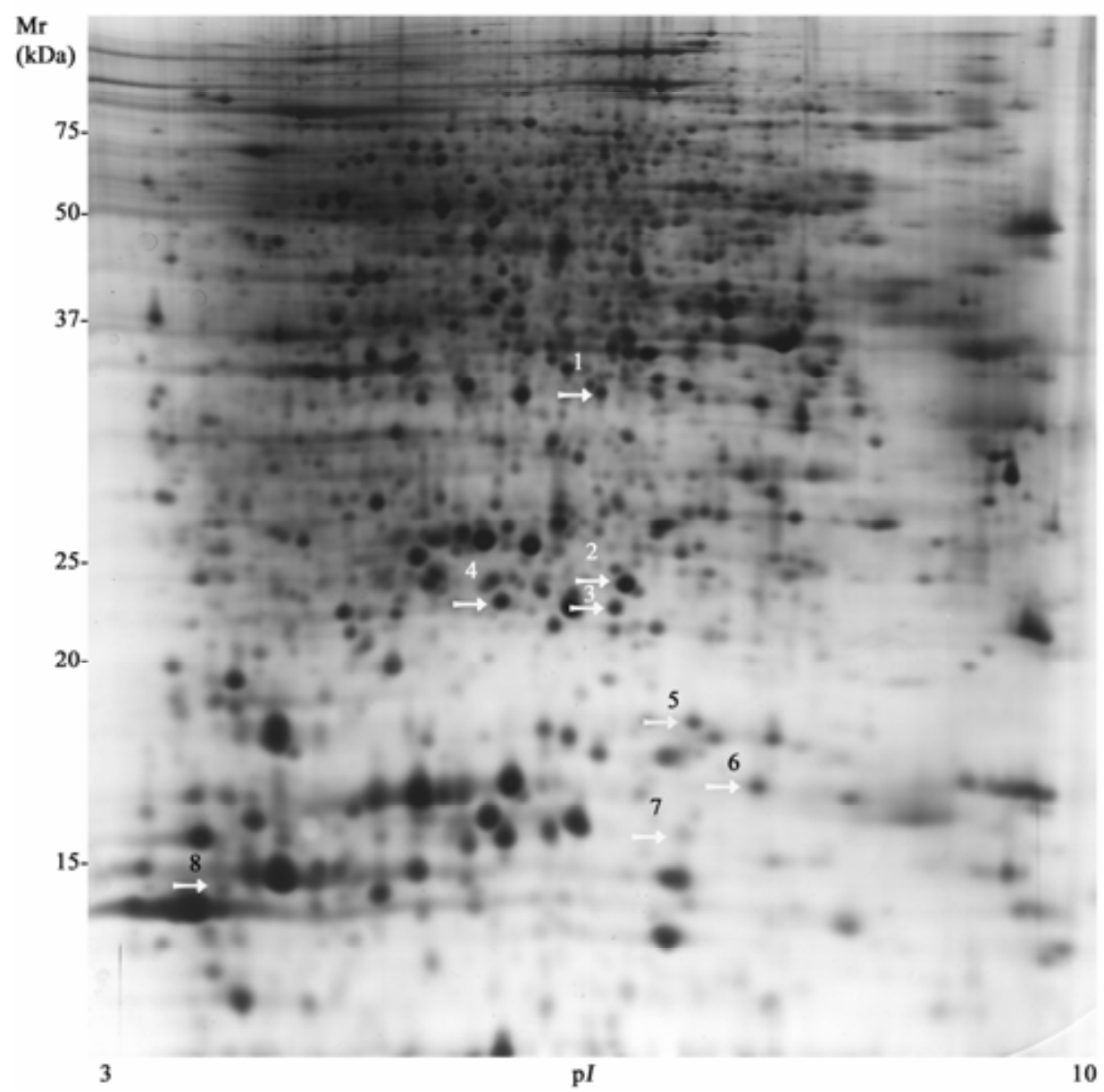

B
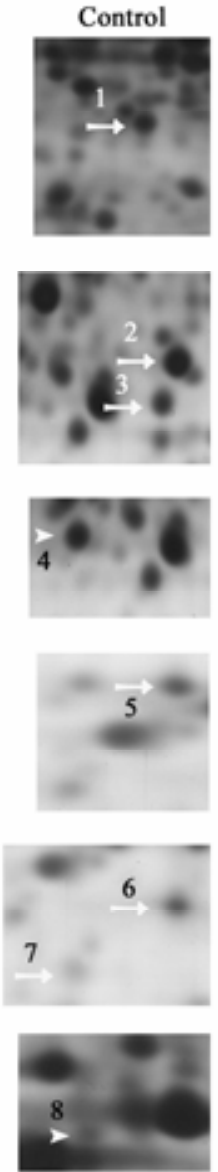

Inoculated
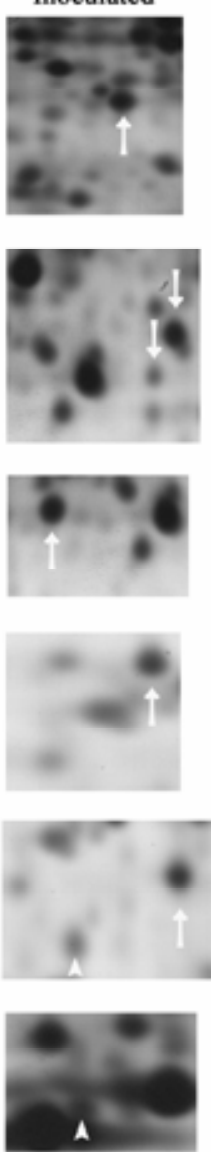

Fig. 1. Comparison of root protein abundance in 5-day-old wild-type (J5) Medicago truncatula plants either Glomus intraradices inoculated or not. A, Twodimensional electrophoretic map of control root proteins and $\mathbf{B}$, changes in protein abundance upon appressorium formation. Horizontal and vertical arrows label root proteins from control and $G$. intraradices-inoculated plants, respectively. Up- and down-headed arrows indicate increase and decrease in root protein abundance in inoculated plants compared with controls, respectively. 
and between experiments for the three $M$. truncatula genotypes, either inoculated or not (data not shown). Consequently, only one representative 2-DE gel or area enlargement is presented for each treatment.

Regarding noninoculated $M$. truncatula plants, proteins from control wild-type roots displayed a pattern, within a window of pI 3 to 10 and molecular mass 10 to $75 \mathrm{kDa}$ (Fig. $1 \mathrm{~A})$, that is consistent with previous proteomic studies carried out with the roots of the noninoculated J5 genotype (Bestel-Corre et al. 2002). Maps very similar to those generated with root proteins from the wild-type genotype were obtained when total proteins were extracted from noninoculated roots of the $M$. truncatula mutants TR122 and TRV25 (Figs. $2 \mathrm{~A}$ and $3 \mathrm{~A}$ ).

For each genotype and each independent experiment, changes in root protein abundance between $G$. intraradicesinoculated and noninoculated plants were quantified by image analysis. Five days after inoculation, the root proteome of the three $M$. truncatula lines was not qualitatively altered upon appressorium formation; however, significant spot volume modifications $(P<0.05)$ were reproducibly quantified from replicated experiments in all genotypes. Changes in protein density in response to $G$. intraradices inoculation are illustrated in Figures 1, 2, and 3 for wild-type (J5), autoregulationdefective (TR122), and mycorrhiza-defective (TR125) genotypes, respectively. Statistically significant differences in root protein accumulation upon appressorium formation were detected for eight spots (spots 1 to 8 ) in the wild-type genotype (Fig. 1), nine spots (spots 2 to 3 and 8 to 14) in the TR122 mutant (Fig. 2), and eight spots (spots 1, 3, 8, and 15 to 19) in the TRV25 mutant (Fig. 3). The constitutive expression of the proteins differentially displayed upon appressorium formation in noninoculated control roots indicated that they were of plant origin.

The groups of proteins that responded to appressorium formation were further compared between wild-type and mutant genotypes. Spots 3 and 8 displayed a common response to $G$. intraradices contact in all genotypes. Upon appressorium formation, spot 1 displayed an increased abundance in wild-type and mycorrhiza-defective genotypes and spot 2 decreased in density in wild-type and hypermycorrhizal genotypes. A genotype-specific response to appressorium formation was observed for four spots (spots 4 to 7 ) in the wild-type line, six spots (spots 9 to 14) in the hypermycorrhizal mutant, and five spots (spots 15 to 19 ) in the mycorrhiza-defective mutant.

\section{Identification of appressorium-responsive proteins.}

The root proteins quantified as differentially displayed in response to $G$. intraradices were excised from micropreparative gels and further analyzed by matrix-assisted laser desorption ionization time-of-flight (MALDI-TOF) mass spectrometry, resulting in 11 identifications (Table 1). All matches were
A

$\mathrm{Mr}$ (kDa)

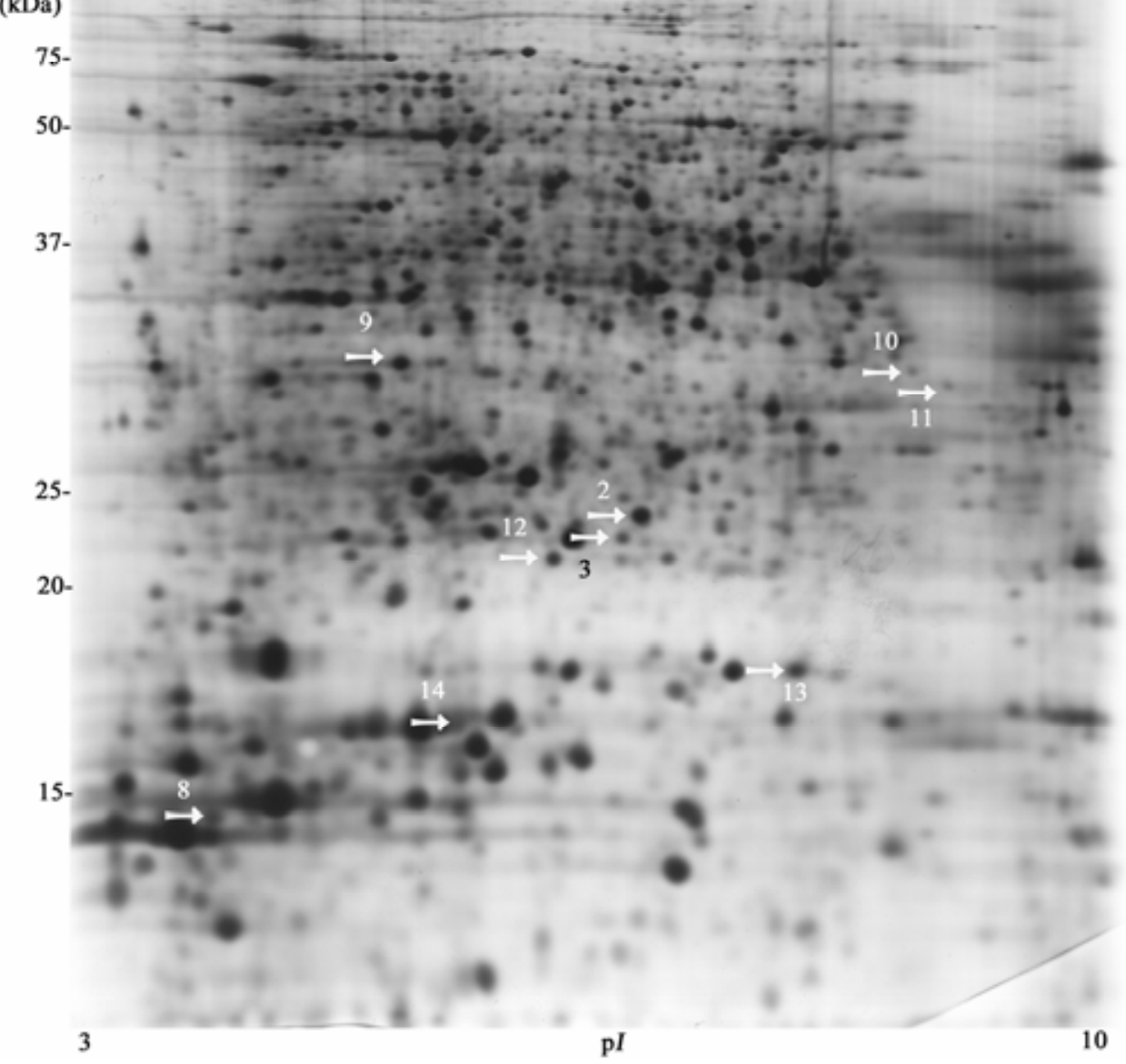

B
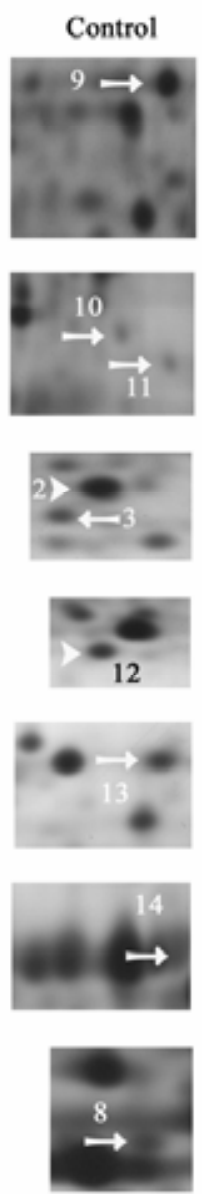

Inoculated
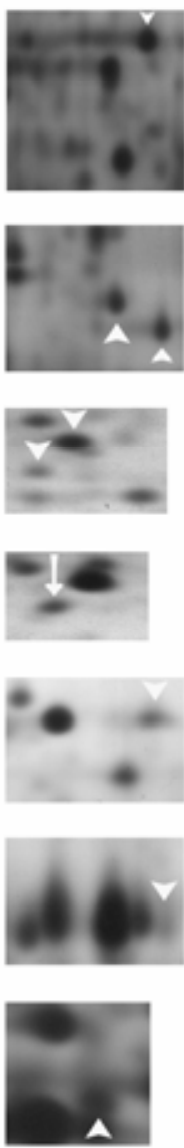

Fig. 2. Comparison of root protein abundance in 5-day-old hypermycorrhizal (TR122) Medicago truncatula plants either Glomus intraradices inoculated or not. A, Two-dimensional electrophoretic map of control root proteins and B, changes in protein abundance upon appressorium formation. Horizontal and vertical arrows label root proteins from control and $G$. intraradices-inoculated plants, respectively. Up- and down-headed arrows indicate increase and decrease in root protein abundance in inoculated plants compared with controls, respectively. 
obtained after peptide mass fingerprinting (PMF) search within the clustered expressed sequence tag (EST) M. truncatula database. Proteins were identified with at least five peptide matches and $21 \%$ sequence coverage. Additionally, the matched peptides overlapped the most intense peaks of the spectra (data not shown) and experimental $\mathrm{pI}$ and $\mathrm{Mr}$ fitted to those calculated, allowing us to ascribe high confidence in the identifications.
A

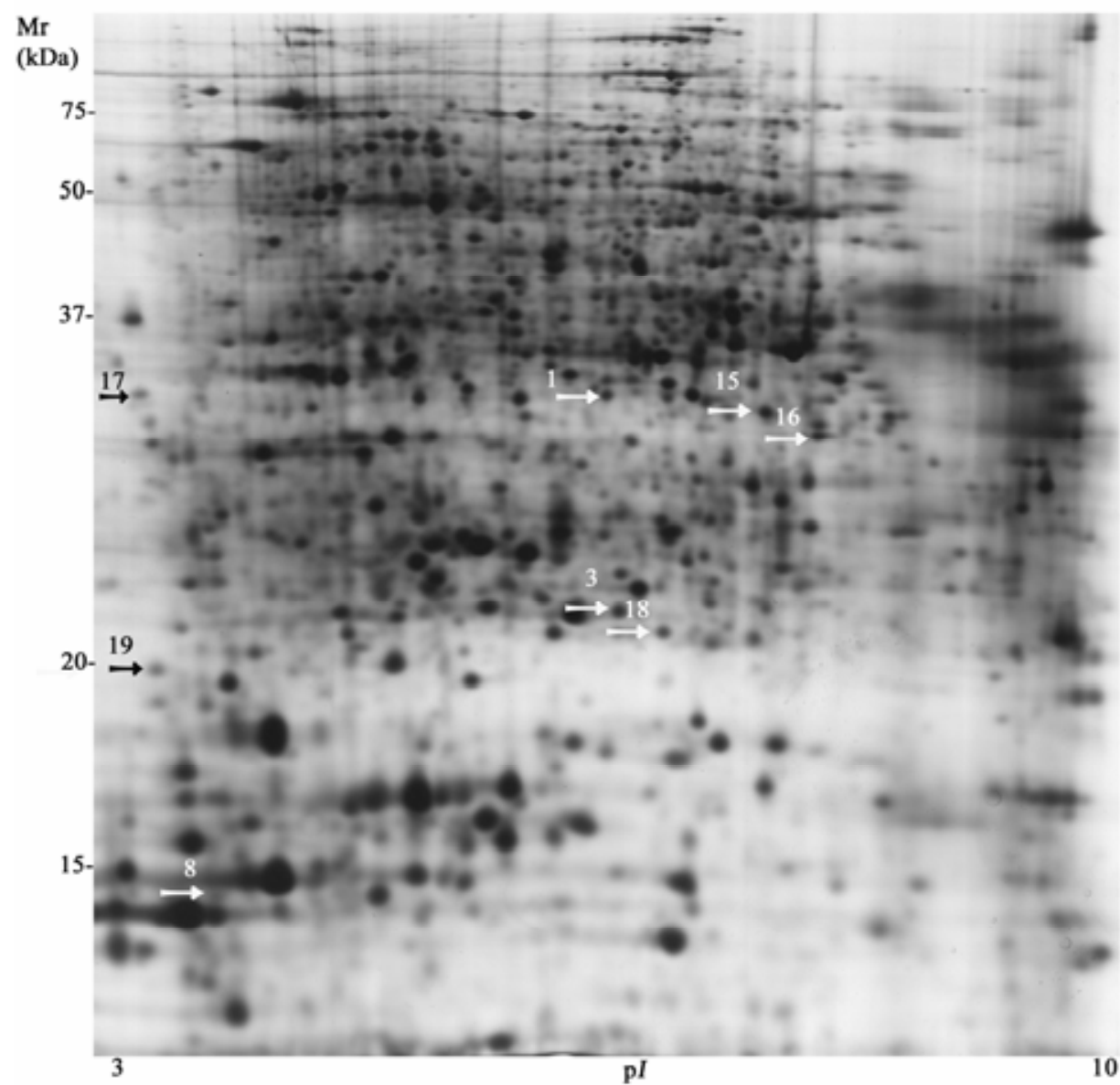

B
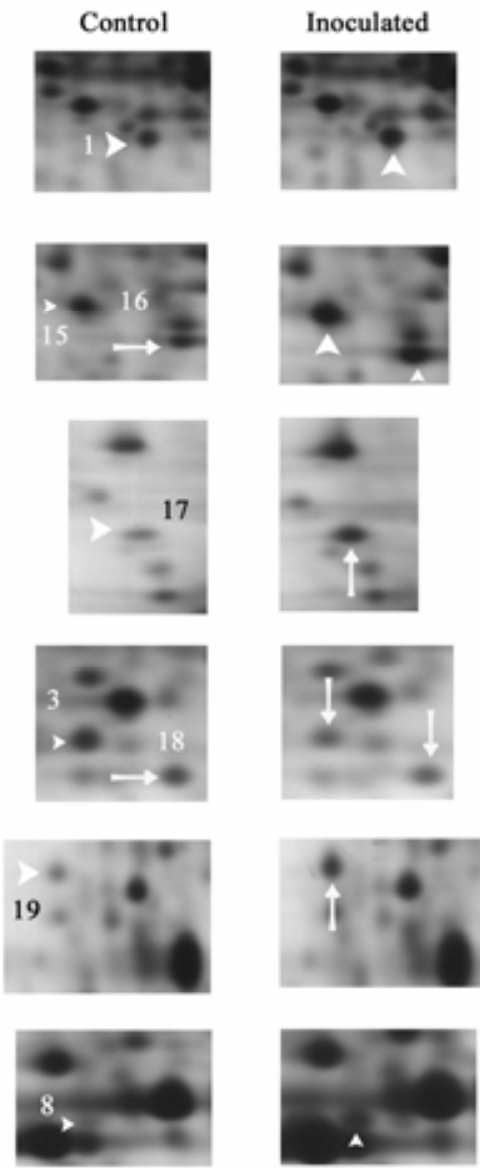

Fig. 3. Comparison of root protein abundance in 5-day-old mycorrhiza-defective (TRV25) Medicago truncatula plants either Glomus intraradices inoculated or not. A, Two-dimensional electrophoretic map of control root proteins and $\mathbf{B}$, changes in protein abundance upon appressorium formation. Horizontal and vertical arrows label root proteins from control and G. intraradices-inoculated plants, respectively. Up- and down-headed arrows indicate increase and decrease in root protein abundance in inoculated plants compared with controls, respectively.

Table 1. Root proteins differentially-displayed upon appressorium formation that could be identified following peptide mass fingerprinting analyses in wildtype (J5), hyper-mycorrhizal (TR122), and mycorrhiza-defective (TRV25) Medicago truncatula plants

\begin{tabular}{|c|c|c|c|c|c|c|c|c|}
\hline $\begin{array}{l}\text { Spot } \\
\text { no. }^{\mathrm{a}}\end{array}$ & Genotype & $\begin{array}{c}\text { Relative } \\
\text { differences }\end{array}$ & $\begin{array}{c}\text { Experimental } \\
\mathrm{Mr} / \mathrm{p} I\end{array}$ & Accession & $\begin{array}{c}\text { No. of } \\
\text { matching } \\
\text { peptides }\end{array}$ & $\begin{array}{c}\text { Coverage } \\
(\%)\end{array}$ & Identification & $\begin{array}{l}\text { Theoretical } \\
\text { Mr/pI }\end{array}$ \\
\hline 1 & $\begin{array}{l}\text { J5 } \\
\text { TRV25 }\end{array}$ & $\begin{array}{r}145(38) \\
87(20)\end{array}$ & $34.64 / 6.78$ & MtC00294 & 10 & 38 & Chalcone reductase & $34.88 / 5.93$ \\
\hline 2 & $\begin{array}{l}\text { J5 } \\
\text { TR122 }\end{array}$ & $\begin{array}{l}-34(2) \\
-57(3)\end{array}$ & $24.41 / 6.93$ & MtC10018 & 20 & 71 & 2,4-D-inducible glutathione transferase & $25.56 / 6.37$ \\
\hline 3 & $\begin{array}{l}\text { J5 } \\
\text { TR122 } \\
\text { TRV25 }\end{array}$ & $\begin{array}{l}-56(8) \\
-41(9) \\
-47(4)\end{array}$ & $23.42 / 6.80$ & MtC10591 & 7 & 33 & Glutathione transferase & $24.38 / 5.99$ \\
\hline 4 & J5 & $121(17)$ & $23.64 / 6.20$ & $\mathrm{MtC} 10060$ & 9 & 39 & $\begin{array}{l}\text { Glutathione-dependent } \\
\text { dehydroascorbate reductase }\end{array}$ & $23.35 / 5.88$ \\
\hline 6 & J5 & $86(4)$ & $18.75 / 7.62$ & MtD20401 & 9 & 40 & Cyclophilin & $18.10 / 8.36$ \\
\hline 7 & J5 & $214(32)$ & $15.62 / 7.18$ & MtC00055 & 7 & 45 & Actin depolymerization factor & $20.83 / 7.68$ \\
\hline 9 & TR122 & $-30(11)$ & $32.10 / 5.68$ & MtC30235 & 19 & 65 & Narbonin & $33.75 / 5.46$ \\
\hline 10 & TR122 & $450(50)$ & $31.20 / 8.30$ & MtC20218 & 5 & 28 & M. truncatula annexin 1 isoform & $34.47 / 8.89$ \\
\hline 11 & TR122 & $308(32)$ & $30.03 / 8.50$ & MtC20218 & 20 & 63 & M. truncatula annexin 1 & $34.47 / 8.89$ \\
\hline 12 & TR122 & $-34(7)$ & $21.51 / 6.55$ & MtD05741 & 5 & 21 & Flavodoxin-like-quinone reductase & $22.09 / 5.88$ \\
\hline 13 & TR122 & $-54(6)$ & $20.02 / 7.70$ & MtC61888 & 10 & 48 & Kunitz-type serine protease inhibitor & $23.41 / 7.61$ \\
\hline
\end{tabular}

${ }^{\text {a }}$ Spots correspond to numbers shown in Figures 1,2 and 3 .

${ }^{\mathrm{b}}$ The difference between spot volumes from mycorrhizal and control treatments was normalized to the volume of the control and expressed in percent. The mean value $(n=4)$ is indicated with the standard deviation in parentheses. 
Among the six root proteins that displayed an increase in abundance in $G$. intraradices-inoculated $\mathrm{J} 5$ plants, a chalcone reductase (spot 1), a glutathione-dependent dehydroascorbate reductase (spot 4), a cyclophilin (spot 6), and an actin depolymerization factor (spot 7) were identified (Table 1). Spots 5 and 8 could not be identified. Proteins that decreased in density upon appressorium formation in $\mathrm{J} 5$ roots corresponded to a 2,4-D-inducible glutathione transferase (spot 2) and a glutathione transferase (spot 3) (Table 1).

In the hypermycorrhizal TR122 mutant, an annexin identical to the M. truncatula annexin 1 (MtAnn1) (spot 10) and one isoform of MtAnn1 (spot 11) belonged to the three proteins that increased in abundance in response to $G$. intraradices (Table 1). Among the six spots that decreased in density upon appressorium formation in the TR122 mutant were the two glutathione transferases (spots 2 and 3) that also displayed a reduced accumulation in wild-type roots, a narbonin (spot 9), a flavodoxin-like quinone reductase (spot 12), and a Kunitz-type serine protease inhibitor (spot 13) (Table 1). Spots 8 and 14 could not be identified.

Among the six proteins that increased in density in response to G. intraradices in the roots of the TRV25 mutant was the chalcone reductase (spot 1) that also displayed an increased abundance upon appressorium formation in wild-type roots (Table 1). The two root proteins that decreased in abundance upon appressorium formation in the TRV25 genotype included the glutathione transferase MtC10591 (spot 3) whose density also was reduced in $G$. intraradices-inoculated J5 and TR122 genotypes (Table 1). Spots 8, 15, 16, 17, 18, and 19 could not be identified.

\section{Identification of common appressorium-responsive proteins between genotypes.}

Upon appressorium formation, an increase in the density of a chalcone reductase was detected in genotypes J5 and TRV25 (Table 1). Belonging to the phenylpropanoid pathway, its activity leads to the formation of the 5-deoxy series of flavonoids and isoflavonoids, which include pterocarpan phytoalexins that act as antimicrobial compounds in Leguminosae (Harborne 1988).

In response to $G$. intraradices, a 2,4-D-inducible glutathione transferase (MtC10018) decreased in density in the hypermycorrhizal mutant TR122 and in the J5 wild-type line (Table 1). Plant glutathione S-transferases (GSTs) form a large family of catalytic and binding proteins that have been associated with stress tolerance, including toxic chemicals, environmental stresses, and diseases (Frova 2003). Among their role as enzymes, they catalyze the addition of the tripeptide glutathione (GSH) to a number of electrophilic compounds and act as a defense mechanism against cellular damage caused by these reactive species. GSTs also act as chaperonins involved in the binding and stabilization of flavonoids and hormones (Marrs 1996). Because of the activation of GST-encoding genes in mature mycorrhiza, it has been proposed that GST expression may be part of a plant defense reaction or involved in arbuscule degradation (Brechenmacher et al. 2004).

In the three genotypes, the abundance of another GST (MtC10591) also decreased in G. intraradices-challenged roots compared with noninoculated ones (Table 1).

\section{Identification \\ of J5-specific appressorium-responsive proteins.}

A GSH-dependent dehydroascorbate reductase (DHAR), belonging to the plant GST superfamily, showed an increase in density in $G$. intraradices-challenged J5 roots compared with noninoculated ones (Table 1). Although displaying the con- served GSH-binding domain, DHARs differ from most other plant GSTs in that they contain a cysteine in place of a serine at the active site and do not have GSH-conjugating activity (Dixon et al. 2002). DHARs catalyze the GSH-dependent reduction of dehydroascorbate to ascorbate, allowing the regeneration of ascorbic acid (vitamin C) that is known as one of the main antioxidant compounds (Blokhina et al. 2003). Antioxidants, such as vitamin C and GSH, prevent peroxidation of lipids and thiol-groups of enzymes and also act as signal-transducing molecules (Foyer et al. 2001).

An increase in the accumulation of a cyclophilin also was detected in the $\mathrm{J} 5$ roots in response to $G$. intraradices inoculation (Table 1). Cyclophilins have an endogenous peptidyl-prolyl cis-trans isomerase (PPIase or romatase) enzymatic activity that directs and accelerates protein folding by catalyzing the isomerization of peptide bonds preceding proline residues (Romano et al. 2005). Cytosolic PPIases have been implicated in a wide range of cellular processes, including the response to abiotic and biotic stresses such as heat shock and pathogen infection (Godoy et al. 2000; Kong et al. 2001; Marivet et al. 1994). A rise in PPIase transcripts also has been reported in yellow lupine upon inoculation with Bradyrhizobium spp. and nodule development (Nuc et al. 2001). Because transcripts mainly accumulated in tissues in which high levels of proline and hydroxyproline-rich proteins were synthesized, it has been suggested that PPIases might be necessary for their accurate folding. Additionally, cyclophilins have a conserved role in signal transduction processes among diverse organisms by participating in the inhibition of phosphatase activities in a $\mathrm{Ca}^{2+}$ dependent manner (Chou and Gasser 1997). A role for cyclophilins in auxin signaling also has been reported (Oh et al. 2006).

Compared with control wild-type roots, an increase in the abundance of an actin depolymerization factor (ADF) was detected in $G$. intraradices-challenged J5 roots (Table 1). ADFs are abundant cytosolic actin-binding proteins involved in the regulation of actin filament turnover that also play a role in signal transduction (Maciver and Hussey 2002). They have been proposed as downstream effectors of calcium-induced actin reorganization that can be phosphorylated by calmodulinlike domain protein kinases (Vantard and Blanchoin 2002).

\section{Identification \\ of TR122-specific appressorium-responsive proteins.}

Upon $G$. intraradices inoculation, the abundance of a narbonin (MtC30235) was decreased in the roots of the autoregulation-deficient mutant (Table 1). Narbonins are predicted to be inactivated chitinases with putative lectin-binding activity (Frosch et al. 2002; Hennig et al. 1995). In Vicia faba, narbonin-like nodulin transcripts, designated VfNOD32, were detected in the nitrogen-fixing zone III of nodules and in much smaller amounts in flowers (Perlick et al. 1996). More recently, decreased levels of the narbonin MtC30235 transcripts have been reported in wild-type and TR122 M. truncatula roots upon inoculation with Sinorhizobium meliloti (El Yahyaoui et al. 2004).

In response to appressorium formation, an increase in the accumulation of MtAnn1 was detected in the root of the hypermycorrhizal mutant (Table 1). MtAnn1, which belongs to a signaling protein family able to bind membrane phospholipids in a calcium-dependent manner, first was characterized as induced by Nod factors and during symbiosis with Rhizobium meliloti (Carvalho-Niebel et al. 1998), pointing to a new early nodulin commonly regulated between mycorrhizal and nitrogen-fixing symbioses. More recently, it has been shown that $M t A n n 1$ expression also was associated with lateral root development and cell differentiation in the root apex independent of 
nodulation (Carvalho-Niebel et al. 2002). Because plant annexins are able to bind to F-actin, it has been suggested that MtAnn1 may play a role in cell cycle reactivation or cytoskeleton rearrangements prior to root infection by rhizobia. Additionally, MtAnn1 displayed increased expression levels in the $R$. meliloti-inoculated roots of the TR122 mutant compared with the wild-type genotype (Carvalho-Niebel et al. 1998).

A decrease in the accumulation of a flavodoxin-like quinone reductase, MtD05741, also was recorded upon appressorium formation in the roots of the TR122 mutant compared with noninoculated ones (Table 1). In most organisms, flavodoxinlike quinone reductases carry out the detoxification of quinones by catalyzing their divalent reduction, then preventing the generation of superoxide radicals and other destructive reactive oxygen species (Ceccarelli et al. 2004). Such oxidants are now well recognized as stimulators of signal transduction under subtoxic conditions, and quinones have been proposed to act as cue molecules through a redox signaling system (Smith et al. 1996). The MtD05741 sequence displayed 76\% amino acid identity with FQR1, a flavodoxin-like quinone reductase from Arabidopsis thaliana that was reported to be a primary auxin-responsive gene. It has been proposed that auxin-inducible quinone reductase acts as a detoxification enzyme to protect plants against auxin-induced oxidative stress (Laskowski et al. 2002).

Upon appressorium formation, the accumulation of a Kunitztype serine protease inhibitor was decreased in the roots of the hypermycorrhizal TR122 mutant (Table 1). One mechanism of plant defense reactions involves a variety of protease inhibitors that can be constitutive or inducible by wounding or pathogen infection (Polya 2001). Many phytopathogenic microorganisms produce extracellular proteases that play a role in pathogenesis, including plant intrusion or inactivation of protective proteins. Plant protease inhibitors are able to suppress enzymatic activity of pathogens and also possess a fungicidal activity (Valueva and Mosolov 2004). Additionally, protease inhibitor proteins can be substrates of calcium-dependent kinases, implying an indirect regulation by $\mathrm{Ca}^{2+}$ fluxes (Polya 2001). In mature mycorrhizal roots, several genes related to encoding plant Kunitztype protease inhibitors have been reported to be upregulated, and promoter activation related to arbuscule development has been demonstrated for MtTi1 (Grunwald et al. 2004).

\section{DISCUSSION}

This study was aimed at investigating changes in the root proteome that were elicited in response to $G$. intraradices appressorium formation in wild-type, mycorrhiza-defective, and autoregulation-defective genotypes of the model legume species M. truncatula. By using a comparative proteomic approach targeted to roots synchronized for appressorium formation, statistically significant changes in protein abundance were recorded between inoculated and noninoculated roots in all genotypes, showing that preinfection triggered root proteome modifications. The use of MALDI-TOF mass spectrometry enabled the first identifications of plant root proteins whose accumulation was altered in response to early stages of AM symbiosis. Except for a chalcone reductase (CHR), none of them previously was known to be appressorium responsive.

The identification in the wild-type genotype of a CHR that increased in abundance prior to root penetration is consistent with the hypothesis that activation of the phenylpropanoid pathway is mediated by early recognition events between the two symbionts (Garcia-Garrido and Ocampo 2002). To our knowledge, changes in the accumulation of GST, GSH-dependent DHAR, cyclophilin (CYP), and ADF proteins upon appressorium formation in roots of mycorrhiza-compatible plants have not been reported previously. The decreased abundance of two GSTs in response to $G$. intraradices suggests that some plant defense reactions may be lowered upon symbiont contact. Remarkably, a role in signal transduction has been proposed for the DHAR, CYP, and ADF that displayed an increased accumulation in $G$. intraradices-inoculated wildtype roots, pointing to the elicitation of new signaling proteins in response to appressorium formation. Although $G$. intraradices differentiated a similar number of appressoria on the roots of the three genotypes 5 days after inoculation, few overlaps but major differences were found when comparing the groups of proteins that responded to appressorium formation between wild-type and mutant genotypes. Despite the fact that some of them could not be identified, this work demonstrated for the first time that mutations in DMI3 and SUNN modified the appressorium-responsive root proteome in AM.

When comparing the root proteins that responded to appressorium formation between wild-type and mycorrhiza-defective $M$. truncatula genotypes, spot 1 (chalcone reductase) also was found to display an increased density in response to $G$. intraradices in the TRV 25 mutant as observed in wild-type roots. This result is consistent with earlier studies that have reported increased levels of chalcone synthase, chalcone isomerase, and phenylalanine ammonia lyase transcripts in the roots of penetration-defective mutants of $M$. truncatula, pea, and alfalfa, respectively, upon inoculation with AM fungi (Bonanomi et al. 2001; Harrison and Dixon 1993; Ruiz-Lozano et al. 1999). In contrast, mutation in DMI3 also was found to trigger modifications in the appressorium-responsive root proteome in that it i) elicited changes in the accumulation of five proteins (spots 15, $16,17,18$, and 19) and ii) modified the response of DHAR, CYP, ADF, 2,4-D-inducible GST proteins, and spot 5 to G. intraradices. This result suggests that the response to $G$. intraradices of proteins 15 to 19 may be repressed by DMI3 at very early stages of AM symbiosis. Unfortunately, spots 15 to 19 could not be identified; therefore, it was not known whether these proteins might play a role or not in the restriction of fungal root penetration in the TRV25 mutant. The fact that DHAR, CYP, ADF, 2,4-D-inducible GST proteins, and spot 5 did not display a response to appressorium formation in the TRV25 mutant compared with the wild-type genotype argued for their DMI3-dependent expression in response to $G$. intraradices. In favor of this conclusion is the role proposed for DHAR, CYP, and ADF in signal transduction mechanisms. Their DMI3-dependent response to $G$. intraradices is consistent with earlier studies that have demonstrated the DMI3-dependent elicitation upon $G$. mosseae appressorium formation of several genes related to signaling pathways (Sanchez et al. 2005; Weidmann et al. 2004). Likewise, a DMI3-dependent change in the accumulation of an actin binding protein (ADF) upon appressorium formation also agrees with the fact that $M$. truncatula dmi mutants failed to develop the early cytoskeleton rearrangements necessary for AM fungus entry (Genre et al. 2005). Additionally, both CYP and ADF proteins have been shown to belong to calcium-modulated signal transduction processes (Chou and Gasser 1997; Vantard and Blanchoin 2002). Their DMI3-dependent response to appressorium formation is consistent with a role for DMI3 in decoding and transducing a calcium spiking signal to downstream responses (Lévy et al. 2004; Mitra et al. 2004). The fact that the decrease in abundance of the 2,4-Dinducible GST in response to $G$. intraradices was not detected in the dmi3 mutant also suggests that this GST may be involved in restricting fungal colonization in the TRV25 genotype.

Unexpected from the TR122 phenotype, which appeared to be different from the wild type only at the arbuscular stage in mycorrhiza, major differences also were observed when comparing the proteins that responded to appressorium formation 
between the wild-type genotype and the autoregulation-defective mutant. They included changes in the abundance of a $\mathrm{CHR}$, a narbonin, two annexins, a flavodoxin-like-quinone reductase (FQR), a protease inhibitor, and DHAR, CYP, and ADF proteins. To our knowledge, this is the first evidence at a proteomic level that $S U N N$ mutation has downstream effects in roots at very early stages of AM symbiosis. This result indicates that differences in protein accumulation occurring prior to root penetration precede the overdevelopment of fungal arbuscules in the hypermycorrhizal mutant. Consequently, the control of arbuscule formation in mycorrhiza (autoregulation of mycorrhization) might be triggered by preinfection events. This conclusion is reminiscent of the autoregulation of nodulation elicited by a prenodulation event (Nod factor induction) (van Brussel et al. 2002). A Nod factor-triggered control of mycorrhization also has been observed (Catford et al. 2003), although it was argued that this result might reflect plant defense reactions elicited by the Nod factor chito-oligosaccharide backbone, rather than true autoregulation (Vierheilig 2004). The $M$. truncatula SUNN gene has been predicted to encode a LRR-RLK (Schnabel et al. 2005); however, so far it is not known how a mutation in $S U N N$ triggers suppression of autoregulation of root symbioses. From the identification of proteins whose response to appressorium formation was modified in the $M$. truncatula sunn mutant, the present study enables us to suggest some clues about the mechanisms by which arbuscular colonization may be controlled at very early stages of AM symbiosis. First, the involvement of defense reactions in the early control of mycorrhization is supported by the fact that the chalcone reductase did not display a density increase upon fungal contact in the TR122 genotype, suggesting that the phenylpropanoid pathway might be differentially regulated in the hypermycorrhizal mutant. Likewise, a decrease in the accumulation of a protease inhibitor was specifically detected in the TR122 genotype in response to appressorium formation. Second, a role for cytoskeleton rearrangement or membrane trafficking events in the early control of mycorrhizal colonization is supported by differences in the abundance of ADF and MtAnn1 proteins between wild-type and hypermycorrhizal genotypes upon appressorium formation. Third, differences in the accumulation of a primary auxin-responsive-like protein (FQR) and a cyclophilin in the roots of the sunn mutant compared with the wild-type genotype suggest a role for auxin signaling in the early control or mycorrhization. This hypothesis is consistent with recent results that have demonstrated that long-distance auxin transport regulation was defective in the M. truncatula sunn mutant (van Noorden et al. 2006). Overall, the identification of signal transduction-related proteins $(F Q R$, MtAnn1, and protease inhibitor) among those displaying a response to appressorium formation specific to the TR122 mutant agrees with the LRR-RLK activity predicted to SUNN. Remarkably, earlier studies have reported increased MtAnnl expression (Carvalho-Niebel et al. 1998) and decreased levels of narbonin, Kunitz-type trypsin inhibitor, and quinone reductase transcripts in the roots of the TR122 mutant upon $S$. meliloti inoculation (El Yahyaoui et al. 2004). Our results point to common early regulatory pathways that may be shared between the two symbioses. The fact that DHAR, ADF, and CYP proteins did not display an increased abundance upon appressorium formation in both dmi3 and sunn mutants compared with the wild-type genotype suggests a role for SUNN at very early stages of AM symbiosis.

To conclude, this study enabled us to identify several plant root proteins that displayed changes in accumulation prior to root penetration by $G$. intraradices. The vast majority of them was not detected previously as appressorium-responsive on the basis of transcriptome analyses, demonstrating that proteomics and transcriptomics are complementary approaches. The results of this work also suggest that SUNN-mediated control of arbuscule formation may be elicited by preinfection events.

\section{MATERIALS AND METHODS}

\section{Plant growth and inoculation.}

Wild-type (J5), mycorrhiza-defective (TRV25, dmi3) (Lévy et al. 2004; Mitra et al. 2004), and hypermycorrhizal (TR122, sunn) (Sagan et al. 1995; Schnabel et al. 2005) genotypes of $M$. truncatula were used in this work. The there genotypes were inoculated or not by the arbuscular mycorrhizal fungus G. intraradices N. C. Schenck \& G. S. Smith (DAOM 181602) according to the following protocol.

Seed were surface sterilized and pregerminated for 2 days as described by Bestel-Corre and associates (2002). For both noninoculated and $G$. intraradices-inoculated plants, six seedlings were transplanted per pot using 38-hole plastic trays. For the three noninoculated genotypes, each $75-\mathrm{ml}$ pot was filled with a sterile mix of Terragreen (charred montmorillonite clay, OilDry-US special type III-R, Mettmann, Germany) and a neutral clay soil from Epoisses (2:1, vol/vol). Mycorrhizal inoculation was performed by replacing the Epoisses soil with a soil-based inoculum (spores, roots, and hyphae) of $G$. intraradices N. C. Schenck \& G. S. Smith (DAOM 181602) produced on leek plants. Seedlings were grown under controlled conditions (16-h photoperiod, 23 and $18^{\circ} \mathrm{C}$ day and night, respectively, $60 \%$ relative humidity, $490 \mu$ Einstein $\mathrm{m}^{-2} \mathrm{~s}^{-1}$ photon lux density) and watered daily with deionized water and weekly with a modified Long Ashton nutritive solution (Dumas-Gaudot et al. 1994). In order to collect plants in which fungal development was restricted to the formation of appressoria at the root surface, pilot experiments were carried out. From 2 to 6 days after inoculation, randomly collected root systems of $G$. intraradices-inoculated plants were cleared with potassium hydroxyde and stained with trypan blue (Phillips and Hayman 1970) to estimate the number of appressoria under a light microscope. As a result, the root samples of $G$. intraradices-inoculated and noninoculated plants were further harvested whatever the plant genotype after 5 days, when no fungal root penetration occurred. For the three genotypes, two replicates of noninoculated and $G$. intraradices-inoculated roots, each consisting of $1 \mathrm{~g}$ (dry weight) of pooled root systems, were frozen in liquid nitrogen and stored at $-80^{\circ} \mathrm{C}$ until protein extraction. The whole experimental design was repeated twice for the three genotypes, resulting in two independent experiments. The absence of rhizobia from the soil-based inocula used in this study was checked visually from the absence of nodules on the roots of additional plants grown until 3 weeks.

\section{Protein extraction.}

Roots from each biological replicate $(1 \mathrm{~g}$ of noninoculated or $G$. intraradices-inoculated roots) were ground in liquid nitrogen and protein extraction was performed as previously described (Dumas-Gaudot et al. 2004). Briefly, ground roots were homogenized in $10 \mathrm{ml}$ of $0.5 \mathrm{M}$ Tris- $\mathrm{HCl}, \mathrm{pH} 7.5$, lysis buffer that contained $0.7 \mathrm{M}$ sucrose, $50 \mathrm{mM}$ EDTA, $0.1 \mathrm{M}$ $\mathrm{KCl}, 10 \mathrm{mM}$ thiourea, $2 \mathrm{mM}$ phenylmethylsulfonyl fluoride, and $2 \%(\mathrm{vol} / \mathrm{vol}) \beta$-mercaptoethanol. One volume of Tris-buffered phenol was added and, after mixing for $30 \mathrm{~min}$, the phenolic phase was separated by centrifugation and rinsed with another $10 \mathrm{ml}$ of lysis buffer. The phenolic phases were pooled and proteins were precipitated overnight at $-20^{\circ} \mathrm{C}$ after adding five volumes of methanol containing $0.1 \mathrm{M}$ ammonium acetate. The pellet, recovered by centrifugation, was rinsed with cold methanol and acetone, dried under nitrogen gas, and solubilized in $400 \mu \mathrm{l}$ of buffer containing $9 \mathrm{M}$ urea, $4 \%$ (wt/vol) 
3-[(3-cholamidopropyl)-dimethyl-ammonio]-1-propanesulfonate (CHAPS), 0.5\% (vol/vol) Triton X-100, $100 \mathrm{mM}$ dithiothreitol (DTT), and 2\% (vol/vol) immobilized $\mathrm{pH}$ gradient (IPG) buffer, pH 3 to 10 (Amersham Biosciences, Little Chalfont, U.K.). Lipids and nucleic acids were removed by ultracentrifugation for 30 min (Beckman Airfuge, $30 \mathrm{psi}$ ) and the protein amount in the supernatant was quantified according to Ramagli and Rodriguez (1985) using ovalbumin as a standard. Samples were stored at $-80^{\circ} \mathrm{C}$ before electrophoresis.

\section{2-DE and image analysis.}

For each biological replicate, at least two 2-DE gels were run. Precast $18-\mathrm{cm}$ nonlinear pH 3 to 10 IPG strips (Amersham Biosciences) were rehydrated overnight with $350 \mu$ of buffer (8 M urea, 2\% [wt/vol] CHAPS, $20 \mathrm{mM} \mathrm{DTT,} \mathrm{2 \%} \mathrm{[vol/vol]}$ IPG Buffer pH 3 to 10, and bromophenol blue) containing 100 $\mu \mathrm{g}$ of proteins. Focusing was performed at $20^{\circ} \mathrm{C}$ for $56 \mathrm{kVh}$ using a gradually increasing voltage. After isoelectric focusing, IPG strips were either stored at $-80^{\circ} \mathrm{C}$ or immediately equilibrated (Görg et al. 1987). The second dimension (sodium dodecyl sulfate polyacrylamide gel electrophoresis) was carried out by transferring the strips onto $12 \%$ acrylamide gels contained in the DALT system (Amersham Biosciences), which allowed both noninoculated and $G$. intraradices-inoculated root protein extracts for each genotype to run simultaneously. Electrophoresis was run at $10^{\circ} \mathrm{C}$ for $1 \mathrm{~h}$ at $30 \mathrm{~V}$, and then at $100 \mathrm{~V}$ for approximately $14 \mathrm{~h}$, until the dye front reached the bottom of the gel. Gels were silver-stained (Mathesius et al. 2001) and digitalized using a Sharp JX-330 scanner (Amersham Biosciences) and the labscan 3.1 software. To monitor differences in protein accumulation between noninoculated and $G$. intraradicesinoculated extracts, gels were analyzed with the Image Master 2D Elite software (Amersham Biosciences). Background subtraction was performed according to the mode of nonspot, and spot volumes were normalized to the total spot volume with a multiplication factor of 100 . For each genotype, difference in spot volumes occurring between the protein profiles of noninoculated and $G$. intraradices-inoculated roots were compared using the Students' $t$ test. Only spots showing statistically significant volume variations $(P<0.05)$ in the two independent experiments were taken into account. Molecular weights and isoelectric points were calculated according to the migration of standard proteins (Bio-Rad, Munich, Germany).

\section{In gel trypsin digestion, MALDI-TOF mass spectrometry, and PMF search.}

The protein spots of interest were excised manually from micropreparative gels in which $500 \mu \mathrm{g}$ of protein had been loaded, and stained with Coomassie Brilliant Blue G 250 (Mathesius et al. 2001). Gel plugs were washed until destaining in $100 \mu \mathrm{l}$ of a $50 \%$ acetonitrile $/ 50 \mathrm{mM}$ hydrogenocarbonate $\mathrm{pH} 8$ solution and then dried under vacuum. After rehydratation in $10 \mu \mathrm{l}$ of $50 \mathrm{mM}$ ammonium hydrogenocarbonate $\mathrm{pH} 8$ containing $0.5 \mu \mathrm{g}$ of porcine trypsin (Promega), samples were incubated overnight ( 16 to $18 \mathrm{~h}$ ) at $37^{\circ} \mathrm{C}$. Peptide masses from digested proteins were obtained using a MALDI-TOF mass spectrometer equipped with an $\mathrm{N}_{2}$ laser $(337 \mathrm{~nm}, 3 \mathrm{~Hz}, 3 \mathrm{~ns}$ impulsion) (Voyager DE super STR; Applied Biosystems, Foster City, CA, U.S.A.). Samples were irradiated in a matrix $(\alpha-$ cyano-4-hydroxycinnamic acid, $3 \mathrm{mg} / \mathrm{ml}$ ) and spectra were acquired in reflectron mode within a 900- to 3,000-Da mass range and a 120-ns delay extraction time. Internal calibration was performed using trypsin peptide masses within a 500- to 5,000-Da mass range.

PMF search was performed on two clustered EST M. truncatula databases (available online) according to Valot and associates (2004). The first, named MtC, contained 6,350 clus- ters defined from three root EST libraries (24,347 ESTs) of a Genoscope project. The clustering process has been previously described by Journet and associates (2002). The second, named $\mathrm{MtD}$, was obtained using the same process on the $M$. truncatula ESTs (approximately 180,000 ESTs) available at the Institute for Genomic Research. It contained 21,400 clusters defined from EST libraries corresponding to different $M$. truncatula tissues.

Protein prospector software was used for PMF search. For peptide matching, a minimum of four peptides matches and $20 \%$ sequence coverage, a maximum of one miscleavage, and peptide modifications by carboxyamidomethylcysteine, methionine sulfoxide, and pyro-glutamic acid or acetylated Nterminal residue were accepted. The maximum tolerance for peptide mass matching was limited to $20 \mathrm{ppm}$.

\section{ACKNOWLEDGMENTS}

The authors are grateful to C. Henry (INRA, Jouy-en-Josas, France) who performed mass spectrometry at the PAPSS platform, G. Duc (INRA, Dijon, France) for providing TR122 and TRV25 seeds, and J. Negrel (INRA, Dijon, France) and M. Parniske for critical reading and helpful suggestions. N. Amiour acknowledges financial support from the Conseil Régional de Bourgogne, Dijon, France.

\section{LITERATURE CITED}

Akiyama, K., Matsuzaki, K., and Hayashi, H. 2005. Plant sesquiterpenes induce hyphal branching in arbuscular mycorrhizal fungi. Nature 435:824-827.

Bestel-Corre, G., Dumas-Gaudot, E., Poinsot, V., Dieu, M., Dierick, J. F., van Tuinen, D., Remacle, J., Gianinazzi-Pearson, V., and Gianinazzi, S. 2002. Proteome analysis and identification of symbiosis-related proteins from Medicago truncatula Gaertn. by two-dimensional electrophoresis and mass spectrometry. Electrophoresis 23:122-137.

Blilou, I., Bueno, P., Ocampo, J. A., and GarciaGarrido, J. 2000. Induction of catalase and ascorbate peroxidase activities in tobacco roots inoculated with the arbuscular mycorrhizal Glomus mosseae. Mycol. Res. 104:722-725.

Blokhina, O., Virolainen, E., and Fagerstedt, K. V. 2003. Antioxidants, oxidative damage and oxygen deprivation stress: A review. Ann. Bot. 91:179-194.

Bonanomi, A., Oetiker, J. H., Guggenheim, R., Boller, T., Wiemken, A., and VogeliLange, R. 2001. Arbuscular mycorrhiza in mini-mycorrhizotrons: First contact of Medicago truncatula roots with Glomus intraradices induces chalcone synthase. New Phytol. 150:573-582.

Brechenmacher, L., Weidmann, S., van Tuinen, D., Chatagnier, O., Gianinazzi, S., Franken, P., and Gianinazzi-Pearson, V. 2004. Expression profiling of up-regulated plant and fungal genes in early and late stages of Medicago truncatula-Glomus mosseae interactions. Mycorrhiza 14:253-262.

Breuninger, M., and Requena, N. 2004. Recognition events in AM symbiosis: Analysis of fungal gene expression at the early appressorium stage. Fungal Genet. Biol. 41:794-804.

Calantzis, C., Morandi, D., Arnould, C., and Gianinazzi-Pearson, V. 2001. Cellular interactions between $G$. mosseae and a Myc(-) dmi2 mutant in Medicago truncatula. Symbiosis 30:97-108.

Carvalho-Niebel, F., Lescure, N., Cullimore, J. V., and Gamas, P. 1998. The Medicago truncatula MtAnnl gene encoding an annexin is induced by Nod factors and during the symbiotic interaction with Rhizobium meliloti. Mol. Plant-Microbe Interact. 11:504-513.

Carvalho-Niebel, F., Timmers, A. C. J., Chabaud, M., Defaux-Petras, A. and Barker, D. G., 2002. The Nod factor-elicited annexin MtAnn1 is preferentially localised at the nuclear periphery in symbiotically activated root tissues of Medicago truncatula. Plant J. 32:343-352.

Catford, J. G., Staehelin, C., Lerat, S., Piche, Y., and Vierheilig, H. 2003. Suppression of arbuscular mycorrhizal colonization and nodulation in split-root systems of alfalfa after pre-inoculation and treatment with Nod factors. J. Exp. Bot. 54:1481-1487.

Catoira, R., Galera, C., de Billy, F., Penmetsa, R. V., Journet, E. P., Maillet, F., Rosenberg, C., Cook, D., Gough, C., and Denarie, J. 2000. Four genes of Medicago truncatula controlling components of a nod factor transduction pathway. Plant Cell 12:1647-1665.

Ceccarelli, E. A., Arakaki, A. K., Cortez, N., and Carrillo, N. 2004. Functional plasticity and catalytic efficiency in plant and bacterial ferre- 
doxin-NADP $(\mathrm{H})$ reductases. Biochim. Biophys. Acta 1698:155-165.

Chou, I. T., and Gasser, C. S. 1997. Characterization of the cyclophilin gene family of Arabidopsis thaliana and phylogenetic analysis of known cyclophilin proteins. Plant Mol. Biol. 35:873-892.

Dixon, D. P., Davis, B. G., and Edwards, R. 2002. Functional divergence in the glutathione transferase superfamily in plants. Identification of two classes with putative functions in redox homeostasis in Arabidopsis thaliana. J. Biol. Chem. 277:30859-30869.

Dumas-Gaudot, E., Guillaume, P., Tahiri-Alaoui, A., Gianinazzi-Pearson, V., and Gianinazzi, S. 1994. Changes in polypeptide patterns in tobacco roots colonized by two Glomus species. Mycorrhiza 4:215-221.

Dumas-Gaudot, E., Valot, B., Bestel-Corre, G., Recorbet, G., St-Arnaud, M., Fontaine, B., Dieu, M., Raes, M., Saravanan, R. S., and Gianinazzi, S. 2004. Proteomics as a way to identify extra-radicular fungal proteins from Glomus intraradices-RiT-DNA carrot root mycorrhizas. FEMS (Fed. Eur. Microbiol. Soc.) Microbiol. Ecol. 48:401-411.

El Yahyaoui, F., Kuster, H., Ben Amor, B., Hohnjec, N., Puhler, A. Becker, A., Gouzy, J., Vernie, T., Gough, C., Niebel, A., Godiard, L., and Gamas, P. 2004. Expression profiling in Medicago truncatula identifies more than 750 genes differentially expressed during nodulation, including many potential regulators of the symbiotic program. Plant Physiol. 136:3159-3176.

Foyer, C. H., Theodoulou, F. L., and Delrot, S. 2001. The functions of inter- and intracellular glutathione transport systems in plants. Trends Plant Sci. 6:486-492.

Frosch, S. C., Becker, J. D., Schultze, M., Schlesier, B., Kahmann, U., Puhler, A., and Perlick, A. M. 2002. Characterization of NVf32: A narbonin-like nodulin of high abundance in Vicia faba L. Plant Sci. 162:401-411.

Frova, C. 2003. The plant glutathione transferase gene family: Genomic structure, functions, expression and evolution. Physiol. Plant 119:469479 .

Garcia-Garrido, J. M., and Ocampo, J. A. 2002. Regulation of the plant defence response in arbuscular mycorrhizal symbiosis. J. Exp. Bot. 53:1377-1386.

Genre, A., Chabaud, M., Timmers, T., Bonfante, P., and Barker, D.G. 2005. Arbuscular mycorrhizal fungi elicit a novel intacellular apparatus in Medicago root epidermal cells before infection. Plant Cell 17:34893799.

Gianinazzi-Pearson, V. 1996. Plant cell responses to arbuscular mycorrhizal fungi: Getting to the roots of the symbiosis. Plant Cell 8:1871-1883.

Giovannetti, M., Sbrana, C., Citernesi, A. S., and Avio, L. 1996. Analysis of factors involved in fungal recognition responses to host-derived signals by arbuscular mycorrhizal fungi. New Phytol. 133:65-71.

Godoy, A. V., Lazzaro, A. S., Casalongue, C. A., and San Segundo, B 2000. Expression of a Solanum tuberosum cyclophilin gene is regulated by fungal infection and abiotic stress conditions. Plant Sci. 152:123134

Görg, A., Postel, W., Weser, J., Günther, S., Strahler, J. R., Hanash, S. M. and Somerlot, L. 1987. Elimination of point streaking on silver stained two-dimensional gels by addition of iodoacetamide to the equilibration buffer. Electrophoresis 8:122-124.

Grunwald, U., Nyamsuren, O., Tarnasloukht, M., Lapopin, L., Becker, A., Mann, P., Gianinazzi-Pearson, V., Krajinski, F., and Franken, P. 2004. Identification of mycorrhiza-regulated genes with arbuscule development-related expression profile. Plant Mol. Biol. 55:553-566.

Harborne, J. B. 1988. Flavonoids in the environment: Structure-activity relationships. Prog. Clin. Biol. Res. 280:17-27.

Harrison, M. J. 1999. Molecular and cellular aspects of the arbuscular mycorrhizal symbiosis. Annu. Rev. Plant Physiol. Plant Mol. Biol. 50:361389

Harrison, M. J., and Dixon, R. A. 1993. Isoflavonoid accumulation and expression of defense gene transcripts during the establishment of vesicular-arbuscular mycorrhizal associations in roots of Medicago truncatula. Mol. Plant-Microbe Interact. 6:643-654

Hennig, M., Pfeffer-Hennig, S., Dauter, Z., Wilson, K. S., Schlesier, B., and Nong, V. H. 1995. Crystal structure of narbonin at 1.8 A resolution. Acta Crystallogr. D Biol. Crystallogr. 51:177-89.

Journet, E. P., van Tuinen, D., Gouzy, J., Crespeau, H., Carreau, V., Farmer, M. J., Niebel, A., Chiex, T., Saillon, O., Chatagnier, O., Godiard, L., Micheli, F., Kahn, D., Gianinazzi-Pearson, V., and Gamas, P. 2002. Exploring root symbiotic programs in the model legume Medicago trunctula using EST analysis. Nucleic Acids Res. 15:5579-5592.

Kong, H., Lee, S., and Hwang, B. 2001. Expression of pepper cyclophilin gene is differentially regulated during the pathogen infection and abiotic stress conditions. Physiol. Mol. Plant Pathol. 59:189-199.

Laskowski, M. J., Dreher, K. A., Gehring, M. A., Abel, S., Gensler, A. L., and Sussex, I. M. 2002. FQR1, a novel primary auxin-response gene, encodes a flavin mononucleotide-binding quinone reductase. Plant Physiol. 128:578-590.
Lévy, J., Bres, C., Geurts, R., Chalhoub, B., Kulikova, O., Duc, G., Journet, E. P., Ane, J. M., Lauber, E., Bisseling, T., Denarie, J., Rosenberg, C., and Debelle, F. 2004. A putative $\mathrm{Ca}^{2+}$ and calmodulin-dependent protein kinase required for bacterial and fungal symbioses. Science 303:1361-1364.

Maciver, S., K., and Hussey, P. J. 2002. The ADF/cofilin family: Actinremodeling proteins. Genome Biol. 3:1-12.

Marivet, J., Margis-Pinheiro, M., Frendo, P., and Burkard, G. 1994. Bean cyclophilin gene expression during plant development and stress conditions. Plant Mol. Biol. 26:1181-1189.

Marrs, K. A. 1996. The functions and regulation of glutathione $S$-transferases in plants. Annu. Rev. Plant Physiol. Plant. Mol. Biol. 47:127158.

Marsh, J. F., and Schultze, M. 2001. Analysis of arbuscular mycorrhizas using symbiosis-defective plant mutants. New Phytol. 150:525-532.

Mathesius, U., Keijzers, G., Natera, S. H., Weinman, J. J., Djordjevic, M. A., and Rolfe, B. G. 2001. Establishment of a root proteome reference map for the model legume Medicago truncatula using the expressed sequence tag database for peptide mass fingerprinting. Proteomics $11: 1424-1440$.

Mitra, R. M., Gleason, C. A., Edwards, A., Hadfield, J., Downie, J. A., Oldroyd, G. E., and Long, S. R. 2004. A Ca ${ }^{2+} /$ calmodulin-dependent protein kinase required for symbiotic nodule development: Gene identification by transcript-based cloning. Proc. Natl. Acad. Sci. USA 101:4701-4705.

Nagahashi, G., and Douds, D. D. 1997. Appressorium formation by AM fungi on isolated cell walls of carrot roots. New Phytol. 136:299-304.

Nuc, K., Nuc, P., and Slomski, R. 2001. Yellow lupine cyclophilin transcripts are highly accumulated in the nodule meristem zone. Mol. PlantMicrobe Interact. 14:1384-1394.

Oh, K., Ivanchenko, M. G., White, T. J., and Lomax, T. L. 2006. The diageotropica gene of tomato encodes a cyclophilin: A novel player in auxin signaling. Planta 4:1-12.

Perlick, A. M., Fruhling, M., Schroder, G., Frosch, S. C., and Puhler, A. 1996. The broad bean gene VfNOD32 encodes a nodulin with sequence similarities to chitinases that is homologous to (alpha/beta)(8)-barreltype seed proteins. Plant Physiol. 110:147-154.

Phillips, J. M., and Hayman, D. S. 1970. Improved procedures for clearing and staining parasite and vesicular-arbuscular mycorrhizal fungi for rapid assessment of infection. Trans. Br. Mycol. Soc. 55:158-161.

Polya, G. 2001. Protease inhibitory plant defensive proteins. Curr. Topics Peptide Protein Res. 4:37-54.

Ramagli, L. S., and Rodriguez, L. W. 1985. Quantification of microgram amounts of protein in two-dimensional polyacrylamide gel electrophoresis sample buffer. Electrophoresis 6:559-563.

Requena, N., Breuninger, M., Franken, P., and Ocon, A. 2003. Symbiotic status, phosphate, and sucrose regulate the expression of two plasma membrane $\mathrm{H}^{+}$-ATPase genes from the mycorrhizal fungus Glomus mosseae. Plant Physiol. 132:1540-1549.

Romano, P., Gray, J., Horton, P., and Luan, S. 2005. Plant immunophilins: Functional versatility beyond protein maturation. New Phytol. 166:752769.

Ruiz-Lozano, J. M., Roussel, H., Gianinazzi, S., and Gianinazzi-Pearson, V. 1999. Defense genes are differentially induced by a mycorrhizal fungus and Rhizobium sp in wild-type and symbiosis-defective pea genotypes. Mol. Plant-Microbe Interact. 12:976-984.

Sagan, M., Morandi, D., Tarenghi, E., and Duc, G. 1995. Selection of nodulation and mycorrhizal mutants in the model plant Medicago truncatula (Gaertn.) after $\gamma$-ray mutagenesis. Plant Sci. 111:63-71.

Sanchez, L., Weidmann, S., Arnould, C., Bernard, A. R., Gianinazzi, S., and Gianinazzi-Pearson, V. 2005. Pseudomonas fluorescens and Glomus mosseae trigger DMI3-dependent activation of genes related to a signal transduction pathway in roots of Medicago truncatula. Plant Physiol. 139:1065-1077.

Schnabel, E., Journet, E.-P., de Carvalho-Niebel, F., Duc, G., and Frugoli, J. 2005. The Medicago truncatula SUNN gene encodes a CLV1-like leucine-rich repeat receptor kinase that regulates nodule number and root length. Plant Mol. Biol. 58:809-822.

Smith, C. E., Ruttledge, T., Zeng, Z., O’Malley, R. C., and Lynn, D. G. 1996. A mechanism for inducing plant development: The genesis of a specific inhibitor. Proc. Natl. Acad. Sci. USA 93:6986-6991.

Smith, S. E., and Read, D. J. 1997. Genetic, cellular and molecular interactions in the establishment of VA mycorrhizas. Pages 81-104 in: Mycorrhizal Symbiosis. S. E. Smith and D. J. Read, eds. Academic Press, London.

Tucker, S. L., and Talbot, N. J. 2001. Surface attachment and pre-penetration stage development by plant pathogenic fungi. Annu. Rev. Phytopathol. 39:385-417.

Valot, V., Dumas-Gaudot, E., and Gianinazzi, S. 2004. Sub-cellular proteomic analysis of a Medicago truncatula root fraction. Phytochemistry 65:1721-1732. 
Valueva, T. A., and Mosolov, V. V. 2004. Role of inhibitors of proteolytic enzymes in plant defense against phytopathogenic microorganisms. Biochemistry 69:1305-1309.

van Brussel, A. A., Tak, T., Boot, K. J., and Kijne, J. W. 2002. Autoregulation of root nodule formation: Signals of both symbiotic partners studied in a split-root system of Vicia sativa subsp. nigra. Mol. PlantMicrobe Interact. 15:341-349.

van Noorden, G. E., Ross, J. J., Reid, J. B., Rolfe, B. G., and Mathesius, U. 2006. Defective long distance signal auxin transport regulation in the Medicago truncatula sunn mutant. 2006. Plant Physiol. 140:14941506.

Vantard, M., and Blanchoin, L. 2002. Actin polymerization processes in plant cells. Curr. Opin. Plant Biol. 5:502-506.

Vierheilig, H. 2004. Regulatory mechanisms during the plant-arbuscular mycorrhizal fungus interaction. Can. J. Bot. 82:1166-1176.
Weidmann, S., Sanchez, L., Descombin, J., Chatagnier, O., Gianinazzi, S. and Gianinazzi-Pearson, V. 2004. Fungal elicitation of signal transduction-related plant genes precedes mycorrhiza establishment and requires the dmi3 gene in Medicago truncatula. Mol. Plant-Microbe Interact. $17: 1385-1393$

\section{AUTHOR-RECOMMENDED INTERNET RESOURCES}

MENS-Medicago EST Navigation System database: medicago.toulouse.inra.fr/Mt/EST/DOC/MtB.html

CNS Genoscope project: www.cns.fr

The Institute for Genomic Research: www.tigr.org/tdb/tgi/mtgi

University of California ProteinProspector software: prospector.ucsf.edu/ucsfhtml4.0/msfit.htm 